Pacific Journal of Mathematics

HOLOMORPHIC FOLIATIONS AND DEFORMATIONS OF THE 


\title{
HOLOMORPHIC FOLIATIONS AND DEFORMATIONS OF THE HOPF FOLIATION
}

\section{T. DUCHAMP AND M. KalKa}

\begin{abstract}
A deformation theory for transversally holomorphic foliations is developed here and used to give an explicit description of the transversally holomorphic foliations near the "Hopf foliations" on odd dimensional spheres.
\end{abstract}

Introduction. In [1] and [2] we began the study of the deformation theory of holomorphic foliations on a smooth compact manifold. Our aim was to construct a reasonably explicit parameterization of a neighborhood of a fixed holomorphic foliation $\mathscr{F}_{0}$ in the space of all foliations by generalizing Kuranishi's theorem on deformations of complex structures on compact complex manifolds. However, in [1] we assumed the existence of a smooth foliation $\mathscr{F}^{\perp}$ transverse to the foliation $\mathscr{F}_{0}$. The purpose of the present paper is to eliminate this rather artificial assumption. In [3] Gomez-Mont observed that the Kodaira-Spencer machine can be used to show the existence of such a parameterization by an analytic subset of a finite dimensional vector space. However, as is the case for the deformation theory of complex structures, the proof is rather abstract and is not easily adapted to computations. To illustrate our results, we present here a classification of all holomorphic foliations near the foliation given by the Hopf fibration $S^{2 n+1} \rightarrow \mathrm{C} P^{n}$.

We shall now give a more precise statement of our results. The reader is assumed to be somewhat familiar with the notations and results of [1]; but we begin with a short review. Let $\mathscr{F}_{0}$ be a fixed holomorphic foliation of real codimensions $2 q$ on the smooth, compact, oriented manifold $M^{n}$, i.e., $\widetilde{F}_{0}$ is given locally by smooth submersions into $\mathbf{C}^{q}$ which patch together via local biholomorphisms of $\mathbf{C}^{q}$. Let $L \subseteq T M$ and $Q=T M / L$ be the (real) tangent and normal bundles of $\mathscr{F}_{0}$ and fix once and for all a splitting $T M=L \oplus Q$ and a Riemannian metric on $M$ respecting it. (In [1] this splitting was assumed to be induced by a transverse foliation. This is not necessarily the case here.) The complex structure map on $Q$ induces a splitting of the complexified normal bundle in the standard way, $Q^{\mathbf{C}}=Q^{(1,0)} \oplus Q^{(0,1)}$ and there is a split exact sequence

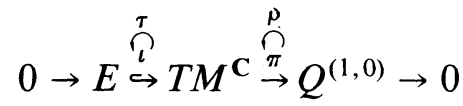


where $E \equiv L^{\mathbf{C}} \oplus Q^{(0,1)}$ and where ( $)^{\mathbf{C}}$ denotes complexification. It will be convenient to identify elements of $E$ and $Q^{(1,0)}$ with either images in $T M^{\mathbf{C}}$; for example, we will sometimes write $X$ for $i(X)$. Finally, set $E_{Q}^{* s}=\Lambda^{s} E^{*}$ $\otimes_{\mathbf{C}} Q^{(1,0)}, s \geq 0$; then $\Gamma\left(E_{Q}^{* s}\right)=\operatorname{Hom}_{\mathbf{C}}\left(\Lambda^{s} E, Q^{(1,0)}\right)$. The ordinary exterior differentiation operator induces operators $d_{Q}: \Gamma\left(E_{Q}^{* s}\right) \rightarrow \Gamma\left(E_{Q}^{* s+1}\right)$ making $\left(\Gamma\left(E^{* s}\right), d_{Q}\right)$ into an elliptic complex [1, page 324]. As usual, let $\delta_{Q}$ be the adjoint of $d_{Q}$, let $\Delta_{Q}$ be the associated Laplacian, let $G_{Q}$ be the Green's operator of $\Delta_{Q}$ and let $H_{Q}$ be the harmonic projection operator. Finally, let $\Theta_{\mathscr{G}}$ denote the sheaf of germs of local holomorphic vector fields, locally constant along the leaves of $\mathscr{F}_{0}$. The complex of sheaves $\left(E_{Q}^{* s}, d_{Q}\right)$ resolves $\Theta_{{\widetilde{\sigma_{0}}}_{0}}$ and by Hodge theory we can identify $H^{s}\left(M, \Theta_{\widetilde{\vartheta}_{0}}\right)$ with the finite dimensional spaces $H_{Q} \Gamma\left(E_{Q}^{* s}\right) \subseteq \Gamma\left(E_{Q}^{* s}\right)$.

Now observe that there is a bijection between maps $\phi \in$ $\operatorname{Hom}\left(E_{Q}, Q^{(1,0)}\right)=\Gamma\left(E_{Q}^{* 1}\right)$ and submodules $E_{\phi} \subseteq T M^{\mathrm{C}}$ near $E=E_{0}$ given by $\phi \mapsto E_{\phi}=\{x+\phi(x) \mid x \in E\}$. It then follows from the complex Frobenius theorem that the subset

$$
\operatorname{Fol}\left(\mathscr{F}_{0}\right)=\left\{\phi \in \Gamma\left(E_{Q}^{* 1}\right) \|\left[\Gamma\left(E_{\phi}\right), \Gamma\left(E_{\phi}\right)\right] \subseteq \Gamma\left(E_{\phi}\right)\right\}
$$

corresponds to a neighborhood of $\mathscr{F}_{0}$ in the space of holomorphic foliations on $M$. In $\S 1$ we shall characterize Fol $\left(\mathscr{F}_{0}\right)$ as the kernel of a certain nonlinear operator

$$
D: \Gamma\left(E_{Q}^{* 1}\right) \rightarrow \Gamma\left(E_{Q}^{* 2}\right)
$$

whose linearization is $d_{Q}$. Our main result follows:

0.3 Theorem A. There is a local analytic set $B \subseteq H^{1}\left(M, \Theta_{\mathscr{F}}\right)$ about $0 \in H^{1}\left(M, \Theta_{\mathscr{F}}\right)$ and a holomorphic map

$$
\Phi:\left\{\begin{array}{l}
B \rightarrow \operatorname{Fol}\left(\mathscr{F}_{0}\right) \subseteq \Gamma\left(E_{Q}^{* 1}\right) \\
\phi_{0} \mapsto \phi\left(\phi_{0}\right)
\end{array}\right.
$$

whose image is a locally complete family of holomorphic foliations near $\mathscr{F}_{0}$. That is, every holomorphic foliation sufficiently near $\mathscr{F}_{0}$ is equivalent, via a diffeomorphism of $M$ near the identity, to an element in the image of $\Phi$. In particular, the space of holomorphic foliations on $M$ is locally path connected. This partially answers a question raised in [6, page 245, Problem 12] concerning the topology of the space of foliations.

As an example, let $M=S^{2 q+1}$ and let $\mathcal{F}_{0}$ be the Hopf foliation given by the standard fibration $S^{2 q+1} \stackrel{\pi}{\rightarrow} \mathrm{C} P^{n}$. Use the holomorphic connection 
$\omega$ on the Hopf bundle to define the splitting $T M=L \oplus Q$ and let the metric on $S^{2 n+1}$ be $g=\omega \otimes \omega+\pi^{*} h$ where $h$ is the Fubini-Study metric on $\mathbf{C} P^{n}$. Because $H^{1}\left(\mathbf{C} P^{n}, \Theta_{\mathbf{C} P^{n}}\right)=0$, it follows from [2] that there is an isomorphism

$$
H^{1}\left(S^{2 q+1}, \Theta_{\widetilde{T}_{0}}\right) \cong \mathbf{C}[\omega] \otimes \Gamma\left(\mathbf{C} P^{q}, \Theta_{\mathbf{C} P^{q}}\right) \cong \Gamma\left(\mathbf{C} P^{n}, \Theta_{\mathbf{C} P^{n}}\right) .
$$

Finally, let $\Omega$ denote the Kähler form on $\mathbf{C} P^{n}$ and observe that $Q^{(1,0)}=$ $\pi^{*} T^{(1,0)} \mathbf{C} P^{n}$ and $E_{Q}^{* 1}=\left(\mathbf{C}[\omega] \oplus \pi^{*} T^{(0,1)} \mathbf{C} P^{q}\right) \otimes \pi^{*} T^{(1,0)} \mathbf{C} P^{q}$.

0.6 THEOREM B. There is an open set $B \subseteq \Gamma\left(\mathbf{C} P^{q}, \Theta_{\mathrm{CP} q}\right)$ about the origin such that the map $\Phi$ in (0.4) is given by the formula

$$
\Phi:\left\{\begin{array}{l}
B \rightarrow\left(E^{*} \otimes Q^{(1,0)}\right) \\
X \mapsto \phi_{X}=-\frac{1}{f_{X}} \omega \otimes X
\end{array}\right.
$$

where $f_{X}$ is a function on $\mathbf{C} P^{n}$ satisfying the conditions

$$
\Omega(X,-)=\bar{\partial} f_{X} .
$$

and

$$
\int_{\mathbf{C} P^{q}} f_{x}^{-1} d \mathrm{vol}=-1
$$

In $\$ 2$ we prove Theorem $B$ and use the fact that the group of biholomorphisms of $\mathbf{C} P^{n}$ is the projective linear group to explicitly compute the image $\Phi$.

REMARK. Since the completion of this paper we learned that Girbau, Haefliger and Sundararaman [3] obtained somewhat stronger results using the Kodaira-Spencer machinery. They compute the Kuranishi family for a class of foliations which includes the Hopf foliation.

1. Proof of Theorem A. We begin with a characterization of the operator $d_{Q}: \Gamma\left(E_{Q}^{* 1}\right) \rightarrow \Gamma\left(E_{Q}^{* 2}\right)$.

1.1 Lemma. Let $\phi \in \Gamma\left(E_{Q}^{* 1}\right)=\operatorname{Hom}\left(E, Q^{(1,0)}\right)$. Then $d_{Q} \phi \in \Gamma\left(E_{Q}^{* 2}\right)$ $=\operatorname{Hom}\left(\Lambda^{2} E, Q^{(1,0)}\right)$ is given by the formula

(1.2) $d_{Q} \phi(X, Y)=\pi([X, \phi(Y)])-\pi([Y, \phi(X)])-\phi \circ \tau([X, Y])$

for all vector fields $X, Y \in \Gamma(E) \subseteq \Gamma\left(T M^{\mathbf{C}}\right)$. 
Proof. This is a straightforward computation after writing $d_{Q} \phi$ in local coordinates adapted to the foliation $\mathscr{F}_{0}$ as in [1, page 320].

Next define operators $[,]_{Q}: \Gamma\left(E_{Q}^{* 1}\right) \times \Gamma\left(E_{Q}^{* 1}\right) \rightarrow \Gamma\left(E_{Q}^{* 2}\right), p_{\tau}: \Gamma\left(E_{Q}^{* 1}\right)$ $\rightarrow \Gamma\left(E_{Q}^{* 2}\right)$ and $D: \Gamma\left(E_{Q}^{* 1}\right) \rightarrow \Gamma\left(E_{Q}^{* 2}\right)$ as follows. For $\phi, \psi \in \Gamma\left(E_{Q}^{* 1}\right)$ and $X$, $Y \in \Gamma(E) \subseteq \Gamma\left(T M^{\mathbf{C}}\right)$, let

$$
\begin{aligned}
{[\phi, \psi]_{Q}(X, Y)=} & \frac{1}{2}\{\phi \circ \tau([X, \psi(Y)])-\phi \circ \tau([Y, \psi(X)]) \\
& +\psi \circ \tau([X, \phi(Y)])-\psi \circ \tau([Y, \phi(X)])\} \\
& -\pi([\phi(X), \psi(Y)])+\pi([\phi(Y), \psi(X)]),
\end{aligned}
$$

and

$$
D \phi=d_{Q} \phi-\left\{[\phi, \phi]_{Q}+p_{\tau}(\phi)\right\} .
$$

We can now characterize holomorphic foliations near $\mathscr{F}_{0}$ as follows:

1.6 Proposition. Given $\phi \in \Gamma\left(E_{Q}^{* 1}\right)$, the associated distribution $E_{\phi}$ defines a holomorphic foliation if and only if $D \phi=0$.

Proof. First observe that a vector $Z \in T M^{\mathrm{C}}$ lies in $E_{\phi}$ if and only if $\pi(Z)-\phi \circ \tau(Z)=0$. Now apply this observation to the vector field $Z=[X+\phi(X), Y+\phi(Y)]$ for $X, Y \in \Gamma(E)$ and conclude that $[X+$ $\phi(X), Y+\phi(Y)] \in \Gamma\left(E_{\phi}\right)$ if and only if $D \phi(X, Y)=0$. The result then follows from the complex Frobenius theorem.

From the above proposition, it follows that the problem of classifying holomorphic foliations near $\mathscr{F}_{0}$ will be solved once we classify the solutions of the equation $D \phi=0$. To do this we need the following estimates.

1.7 Lemma. Let $\phi, \psi \in \operatorname{Hom}\left(E, Q^{(1,0)}\right)$. Then

$$
\left\|[\phi, \psi]_{Q}\right\|_{s} \leq C\|\phi\|_{s+1}\|\psi\|_{s+1}
$$

and

$$
\left\|p_{\tau}(\phi)-p_{\tau}(\psi)\right\|_{s} \leq C\|\phi-\psi\|_{s}\left(\|\phi\|_{s}+\|\psi\|_{s}\right)^{2} .
$$

We have used the notation \|\|$_{s}$ for the usual Sobolev norms on sections of the relevant bundles.

Proof. The easiest way to see this is to write out explicit local formulae for these operators. In coordinates adapted to the foliation $\mathscr{F}_{0}$, 
let $\left\{\partial / \partial x^{i}, \partial / \partial z^{\alpha}\right\}$ span $E$ and $\left\{\left[\partial / \partial z^{\alpha}\right]\right\}$ span $Q^{(1,0)}$. Label elements of $\left\{\partial / \partial x^{i}, \partial / \partial z^{\alpha}\right\}$ by $Y_{J}$ and their duals by $Y^{j^{*}}$, and set $\left[\partial / \partial z^{\alpha}\right]=Z_{\alpha}$. Here $i$ ranges between 1 and $p$ and $\alpha$ between 1 and $q, p=\operatorname{dim} L, q=\operatorname{dim} Q^{(0,1)}$. Then, if we set $\tau\left[Z_{\alpha}, Z_{\beta}\right]=C_{\alpha \beta}^{j} Y_{j}$ and $\tau\left[Y_{l}, Z_{\alpha}\right]=D_{l \alpha}^{s} Y_{s}$, we have for

$$
\phi=\phi_{J}^{\alpha}\left(Y^{j^{*}} \otimes Z_{\alpha}\right)
$$

and

$$
\psi=\psi_{j}^{\alpha}\left(Y^{j^{*}} \otimes Z_{\alpha}\right)
$$

the formula

$$
\begin{aligned}
{[\phi, \psi]_{Q}=\left[\frac{1}{2}\{\right.} & \phi_{l}^{\alpha} Z_{\alpha}\left(\psi_{s}^{\beta}\right)+\psi_{s}^{\alpha} Z_{\alpha}\left(\phi_{l}^{\beta}\right) \\
& \left.\left.+\left(\phi_{t}^{\beta} \psi_{s}^{\alpha}+\psi_{t}^{\beta} \phi_{s}^{\alpha}\right) D_{l \alpha}^{t}\right\}\right] Y^{* l} \wedge Y^{* s} \otimes Z_{\beta} .
\end{aligned}
$$

Inequality (1.8) now follows. With the same notation we see that

$$
p_{\tau}(\phi)=\left(C_{\alpha \beta}^{J} \phi_{j}^{\delta} \phi_{l}^{\alpha} \phi_{s}^{\beta}\right) Y^{* l} \wedge Y^{* s} \otimes Z_{\delta} .
$$

So we see that

$$
\begin{aligned}
p_{\tau}(\phi)-p_{\tau}(\psi) & =C_{\alpha \beta}^{J}\left(\phi_{j}^{\delta} \phi_{l}^{\alpha} \phi_{s}^{\beta}-\psi_{J}^{\delta} \psi_{l}^{\alpha} \psi_{s}^{\beta}\right) Y^{* l} \wedge Y^{* s} \otimes Z_{\delta} \\
= & C_{\alpha \beta}^{J}\left\{\left(\phi_{J}^{\delta}-\psi_{J}^{\delta}\right) \phi_{l}^{\alpha} \psi_{s}^{\beta}+\psi_{j}^{\delta}\left(\phi_{l}^{\alpha}-\psi_{l}^{\alpha}\right) \phi_{s}^{\beta}+\psi_{J}^{\delta} \psi_{l}^{\alpha}\left(\phi_{s}^{\beta}-\psi_{s}^{\beta}\right)\right\} \\
& \wedge Y^{* s} \otimes Z_{\delta} .
\end{aligned}
$$

Inequality (1.9) follows from this formula.

1.12 Remark. Observe that $p_{\tau}$ depends on the splitting (0.1). Suppose that the splitting satisfies the integrability condition $\left[\Gamma\left(Q^{(1,0)}\right), \Gamma\left(Q^{(1,0)}\right)\right]$ $\subseteq \Gamma\left(Q^{(1,0)}\right)$. Then $p_{\tau} \equiv 0$ as can be seen from (1.11) and the fact that $C_{\alpha \beta}^{l} \equiv 0$. If $(0.1)$ is induced by a foliation transverse to $\mathscr{F}_{0}$, or if $\mathscr{F}_{0}$ is given by the fibers of a holomorphic fiber bundle and (0.1) is induced by a holomorphic connection, then the above integrability condition is satisfied.

The next proposition shows that we need not examine all solutions of the equation $D \phi=0$.

1.13 Proposition. Every solution of the equation $D \phi=0$ of sufficiently small norm is equivalent, via a diffeomorphism close to the identity, to a solution of the system

$$
\left\{\begin{array}{c}
D \phi=0 \\
\delta_{Q} \phi=0 .
\end{array}\right.
$$


Proof. The argument in [1, pp. 330-334] applies here. Simply replace the local vector fields $\partial / \partial z^{\alpha}$ in $[1]$ by the fields $Z_{\alpha} \equiv\left[\partial / \partial z^{\alpha}\right]$. Nowhere in the argument was the integrability of the splitting (0.2) used.

To investigate solutions of (1.14) we need the following lemma.

1.15 Lemma. For $\phi_{0} \in H_{Q} \Gamma\left(E_{Q}^{* 1}\right)$ of sufficiently small norm, say $\varepsilon$, there is a unique solution $\phi=\phi\left(\phi_{0}\right)$ of the equation

$$
\phi=\phi_{0}+\delta_{Q} G_{Q}\left\{[\phi, \phi]_{Q}+p_{\tau}(\phi)\right\} \text {. }
$$

Moreover, the map $\phi_{0} \mapsto \phi\left(\phi_{0}\right)$ is holomorphic.

Proof. We solve the equation by iteration, using the estimates of Lemma 1.7 together with the standard elliptic estimate $\left\|G_{Q} \phi\right\|_{s} \leq C\|\phi\|_{s-2}$.

For $n \geq 0$, set $\phi_{n+1}=\phi_{0}+d_{Q}^{*} G_{Q}\left\{\left[\phi_{n}, \phi_{n}\right]_{Q}+p_{\tau}\left(\phi_{n}\right)\right\}$. Then

$$
\begin{aligned}
& \left\|\phi_{n+1}-\phi_{n}\right\|_{s} \\
& \quad=\left\|d_{Q}^{*} G_{Q}\left\{\left[\phi_{n}, \phi_{n}\right]_{Q}-\left[\phi_{n-1}, \phi_{n-1}\right]_{Q}+p_{\tau}\left(\phi_{n}\right)-p_{\tau}\left(\phi_{n-1}\right)\right\}\right\|_{s} \\
& \leq C\left\|\left[\phi_{n}, \phi_{n}\right]_{Q}-\left[\phi_{n-1}, \phi_{n-1}\right]_{Q}+p_{\tau}\left(\phi_{n}\right)-p_{\tau}\left(\phi_{n-1}\right)\right\|_{s-1} \\
& \leq C\left\{\left\|\left[\phi_{n}, \phi_{n}\right]_{0}-\left[\phi_{n-1}, \phi_{n-1}\right]_{Q}\right\|_{s-1}+\left\|p_{\tau}\left(\phi_{n}\right)-p_{\tau}\left(\phi_{n-1}\right)\right\|_{s-1}\right\} \\
& \leq C\left\{\left\|\left[\phi_{n}-\phi_{n-1}, \phi_{n}+\phi_{n-1}\right]_{Q}\right\|_{s-1}\right. \\
& \left.\quad+\left\|\phi_{n}-\phi_{n-1}\right\|_{s-1} \max \left(\left\|\phi_{n}\right\|_{s-1},\left\|\phi_{n-1}\right\|_{s-1}\right)\right\} \\
& \leq C\left\|\phi_{n}-\phi_{n-1}\right\|_{s}\left\{\left\|\phi_{n}\right\|_{s}+\left\|\phi_{n-1}\right\|_{s}+\max \left(\left\|\phi_{n}\right\|_{s+1},\left\|\phi_{n-1}\right\|_{s-1}\right)\right\} .
\end{aligned}
$$

Thus for $\left\|\phi_{0}\right\|_{C^{\infty}}<1 / 3 C, \phi_{n}$ converges in $H^{s}$ for all $s$.

Uniqueless follows in a similar fashion. Suppose $\phi, \psi$ are two solutions. Then

$$
\begin{aligned}
\|\phi-\psi\|_{s} & =\left\|\delta_{Q} G\left([\phi, \phi]_{Q}-[\psi, \psi]_{Q}\right)+p_{\tau}(\phi)-p_{\tau}(\psi)\right\|_{s} \\
& \leq C\|\phi-\psi\|_{s}\left\{\|\phi\|_{s}+\|\psi\|_{s}\right\} .
\end{aligned}
$$

Thus, for $\left\|\phi_{0}\right\|_{s}$ small, the solution is unique. Holomorphic dependence on $\phi_{0}$ is standard, for example, [5].)

We can now define the analytic subset $B \subseteq H_{Q} \Gamma\left(E_{Q}^{* 1}\right)=H^{1}\left(M, \Theta_{\widetilde{\vartheta}_{0}}\right)$ of Theorem A. A section $\phi_{0}$ is in $B$ if the following conditions are satisfied:

$$
\begin{gathered}
\left\|\phi_{0}\right\|_{C^{\infty}}<\varepsilon, \\
H_{Q}\left\{\left[\phi\left(\phi_{0}\right), \phi\left(\phi_{0}\right)\right]_{Q}+p_{\tau} \phi\left(\phi_{0}\right)\right\}=0,
\end{gathered}
$$


and

$$
d_{Q}\left\{\left[\phi\left(\phi_{0}\right), \phi\left(\phi_{0}\right)\right]_{Q}+p_{\tau} \phi\left(\phi_{0}\right)\right\}=0 .
$$

The following proposition concludes the proof of Theorem A.

1.20 Proposition. $\phi$ is a solution of (1.14) with $\|\phi\|_{C^{\infty}}<\varepsilon$ if and only if it is in the image of the map

$$
\left\{\begin{array}{l}
B \rightarrow \operatorname{Fol}\left(\mathscr{F}_{0}\right) \\
\phi_{0} \mapsto \phi\left(\phi_{0}\right)
\end{array} .\right.
$$

Proof. $\phi$ is a solution of (1.14) if and only if the following equations are satisfied:

$$
\delta_{Q} \phi=0,
$$

$$
\begin{gathered}
H_{Q}\left\{d_{Q} \phi-\left([\phi, \phi]_{Q}+p_{\tau}(\phi)\right)\right\}=-H_{Q}\left([\phi, \phi]+p_{\tau}(\phi)\right)=0, \\
d_{Q}\left([\phi, \phi]_{Q}+p_{\tau}(\phi)\right)=0
\end{gathered}
$$

and

$$
\delta_{Q}\left(d_{Q} \phi-\left\{[\phi, \phi]_{Q}+p_{\tau}(\phi)\right\}\right)=0 .
$$

In particular,

$$
\delta_{Q}\left(d_{Q} \phi-\left\{[\phi, \phi]_{Q}+p_{\tau}(\phi)\right\}\right)=0
$$

and

$$
d_{Q} \delta_{Q} \phi=0
$$

Adding, we get the equation

$$
\Delta_{Q} \phi-\left\{[\phi, \phi]_{Q}+p_{\tau}(\phi)\right\}=0 .
$$

If we apply the Green's operator $G_{Q}$ to the last equation, and use the identity $G_{Q} \Delta_{Q}=$ Id $-H_{Q}$, we get the equation

$$
\phi=H_{Q} \phi+\delta_{Q} G_{Q}\left\{[\phi, \phi]_{Q}+p_{\tau}(\phi)\right\} \text {. }
$$

Setting $\phi_{0}=H_{Q} \phi$ proves the proposition.

REMARK. In [1] we were able to simplify the form that $B$ takes, and use this to conclude that if $H^{2}\left(M, \Theta_{\mathscr{F}}\right)=0$, then $B=$ a neighborhood of 0 in $H^{1}\left(M, \Theta_{\mathscr{F}}\right)$. The reason that we cannot make the same conclusion here is that the operator $[,]_{Q}+p_{\tau}$ does not have the pleasant algebraic properties (2.12)-(2.14) of [1], unless the splitting (0.2) is integrable. See 
Remark 1.12. In case $\left[\Gamma\left(Q^{1,0}\right), \Gamma\left(Q^{(1,0)}\right] \subset \Gamma\left(Q^{1,0}\right)\right.$, then our method allows us to conclude that $H^{2}\left(M, \Theta_{\mathscr{F}}\right)=0 \Rightarrow B=$ a neighborhood of 0 in $H^{1}\left(M, \Theta_{\mathscr{f}}\right)$. However, in [3] it is shown that $H^{2}\left(M, \Theta_{\mathscr{f}}\right)=0$ implies $B=$ a neighborhood of 0 in $H^{1}\left(M, \Theta_{\mathscr{\sigma}}\right)$.

2. Deformations of the Hopf foliation. As an application of Theorem A, we now consider the holomorphic foliations on $M=S^{2 q+1}=$ $\left\{\left(z^{0}, \ldots, z^{q}\right) \in \mathbf{C}^{q+1} \mid \sum_{\alpha=0}^{q} z^{\alpha} \bar{z}^{\alpha}=1\right\}$ near the holomorphic foliation $\mathscr{F}_{0}$ given by the Hopf map $\pi: S^{2 q+1} \rightarrow \mathbf{C P} P^{q}$. The notation is as in the introduction. Via the projection map $\pi$ we can make the identifications $\Gamma\left(\Theta_{\mathbf{C} P^{q}}\right)=\Gamma\left(\Theta_{\mathscr{F}_{0}}\right)$ and $C^{\infty}\left(\mathbf{C} P^{q}\right) \subseteq C^{\infty}\left(S^{2 q+1}\right)$.

We first compute $H_{Q} \Gamma\left(E_{Q}^{* 1}\right) \subseteq \operatorname{Hom}\left(E, Q^{(1,0)}\right)$.

2.1 LEMMA. Let $\mathrm{g}$ be a $\mathrm{C}^{\infty}$-function on $\mathrm{CP}^{q}$ and $X$ a holomorphic vector field on $\mathbf{C} P^{q}$. Then

$$
d_{Q}(\omega \otimes X)=0
$$

and

$$
\delta_{Q}(g \omega \otimes X)=0 .
$$

In particular, $H_{0} \Gamma\left(E_{Q}^{* 1}\right)=\left\{\omega \otimes X \mid X \in \Gamma\left(\Theta_{\mathbf{C} P^{Q}}\right)\right\}$.

Moreover,

$$
H_{Q}(g \omega \otimes X)=\omega \otimes(a X)
$$

where $a=\int_{\mathbf{C} P^{q}} g \nu$ and $\nu=\Omega^{q} /(q !)$.

Proof. Let $p_{*}: \Lambda T^{*} M^{\mathbf{C}} \rightarrow \Lambda E^{*}$ be the natural mapping. Then $d_{Q}(\omega \otimes X)=\left(p_{*} d \omega\right) \otimes X=2 \pi p_{*}\left(\pi^{*} \Omega\right) \otimes X=0$ since $d \omega=2 \pi\left(\pi^{*} \Omega\right)$ and since $\Omega$ is of type $(1,1)$.

To verify (2.3) we must show that for $Y \in \Gamma\left(Q^{(1,0)}\right)$ the equation $\left\langle d_{Q} Y, g \omega \otimes X\right\rangle=0$ is satisfied. Write $Y$ in the form $Y=\Sigma_{i} f_{i} X_{\imath}$ for $f_{i}$ smooth functions on $S^{2 q+1}$ and $X_{i}$ holomorphic vector fields on $\mathbf{C} P^{q}$. Then a standard computation using the formulas (1.17) and (1.30) of [1] together with Stokes' Theorem yields:

$$
\begin{aligned}
\left\langle d_{Q} Y, g \omega \otimes X\right\rangle & =\sum_{i} \int_{S^{2 q+1}}\left(d f_{i}\right) \wedge g \cdot h\left(X_{i}, X\right) \nu \\
& =\sum_{i} \int_{S^{2 q+1}} d\left(f_{i} g h\left(X_{i}, X\right) \nu\right)=0 .
\end{aligned}
$$


Finally, to verify (2.4), a set $\phi_{0}=\omega \otimes a X$ and let $\bar{\partial} *$ be the adjoint of the $\bar{\partial}$ operator on the complex of $T^{1,0}\left(\mathbf{C} P^{q}\right)$-valued $(0, p)=$ forms on $\mathbf{C} P^{q}$. It follows that $(g-a) X=\bar{\partial}^{*} \eta$ for $\eta$ a $T^{1,0}\left(\mathbf{C} P^{q}\right)$-valued $(0,1)$-form. Now let $\psi=\omega \otimes Y$ be a harmonic element of $\Gamma\left(E^{* 1}\right)$ where $Y \in \Gamma\left(\Theta_{\mathbf{C} P^{q}}\right)$. Then the result follows from the computation:

$$
\begin{aligned}
\left\langle\psi, \phi-\phi_{0}\right\rangle & =\int_{S^{2 q+1}} \omega \wedge(\overline{g-a}) h(Y, X) \nu \\
& =\int_{\mathbf{C} P^{q}}\left[\int_{\text {fiber }} \omega\right](\overline{g-a}) h(Y, X) \nu \\
& =2 \pi \int_{\mathbf{C} P^{q}}(\overline{g-a}) h(Y, X) \nu=2 \pi\langle Y,(g-a) X\rangle_{\mathbf{C} P^{q}} \\
& =2 \pi\left\langle Y, \bar{\partial}^{*} \eta\right\rangle_{\mathbf{C} P^{q}}=2 \pi\langle\bar{\partial} Y, \eta\rangle_{\mathbf{C} P^{q}}=0
\end{aligned}
$$

since $\bar{\partial} Y=0$.

Proof of Theorem B. By the results of $\S 1$, every holomorphic foliation near $\mathscr{F}_{0}$ is equivalent to one of the form $E_{\phi}$ where $\phi$ satisfies the equations

$$
\begin{gathered}
D \phi=0, \\
\delta_{Q} \phi=0,
\end{gathered}
$$

and

$$
H_{Q} \phi=\phi_{0}
$$

for $\phi_{0}=\omega \otimes X$ and $X \in \Gamma\left(\Theta_{\mathbf{C} P^{q}}\right)$. Moreover, the section $\phi_{0}$ completely determines $\phi$. It is reasonable (and correct) to try $\phi=g \omega \otimes X$ for $g$ a function of $\mathbf{C} P^{q}$ satisfying the condition $\int_{\mathbf{C} P^{q}} g \boldsymbol{\nu}=1$. By the above lemma, (2.6) and (2.7) will hold true.

By Remark 1.12, $p_{\tau} \equiv 0$ so to solve (2.5) we must compute $[\phi, \phi]_{Q}$ :

$$
\begin{aligned}
{[\phi, \phi]_{Q}(U, V) } & =\phi([\phi(U), V])-\phi([\phi(V), U])-\pi[\phi(U), \phi(V)] \\
& =\left\{\begin{array}{l}
0 \quad \text { if } U, V \text { are both horizontal or both vertical } \\
g \omega([g \omega(U) X, V]) X=-g^{2} \omega(U) \Omega(X, V) X \text { if } \\
U \text { is vertical and } V \text { is horizontal. }
\end{array}\right.
\end{aligned}
$$

Thus,

$$
d_{Q} \phi-[\phi, \phi]_{Q}=\bar{\partial} g \wedge \omega \otimes X+g^{2} \omega \wedge \Omega(X,-) \otimes X
$$

and $\phi$ defines a holomorphic foliation iff this expression is zero. Thus we need to find $g$ satisfying $\bar{\partial} g-g^{2} \Omega(X,-)=0$. Since $H^{1}\left(\mathbf{C P}^{n}, \theta\right)=0$, 
$\Omega(X,-)=\bar{\partial} f_{X}$, for some $f_{X}$, unique up to a constant. Thus we need to solve the equation

$$
\bar{\partial} g=g^{2} \bar{\partial} f_{X}
$$

which has $g_{X}=-1 / f_{X}$ as a solution. By choosing $X$ sufficiently small and fixing $f_{X}$ by the normalization $\int_{\mathbf{C} P^{q}} g_{X} \nu=1$, we obtain the required solution.

We shall now utilize Theorem $B$ to give a more concrete parameterization of the set of holomorphic foliations near the Hopf foliation.

Let $z=\left(z^{0}, \ldots, z^{q}\right)$ be a point in $\mathbf{C}^{q+1}$, let $[\mathbf{z}]=\left[z^{0}, \ldots, z^{q}\right]$ be the associated point in $\mathbf{C} P^{q}$ and let $|z|=\sqrt{z^{\alpha} \bar{z}}{ }^{\alpha}$. Greek indices will range from 0 to $q$ in this section and the summation convention is in effect throughout. Identify $S^{2 q+1}$ with the unit sphere $\left\{|z|^{2}=1\right\}$. Then $\omega$ is the restriction to $S^{2 q+1}$ of the form $\bar{\partial}|z|^{2}$ and $\Omega=i \partial \bar{\partial} \log |z|^{2}$. Note that the vertical vector field on $S^{2 q+1} \rightarrow \mathrm{C} P^{q}$ is the vector field

$$
V=i / 2\left(z^{\alpha} \frac{\partial}{\partial z^{\alpha}}-\bar{z}^{\alpha} \frac{\partial}{\partial \bar{z}^{\alpha}}\right)
$$

Now every holomorphic vector field on $\mathbf{C} P^{q}$ arises in the following way: Choose a complex matrix $A=\left(a_{\alpha}^{\beta}\right) \in \mathrm{GL}(q+1, \mathbf{C})$ and set

$$
\tilde{X}_{A}=a_{\alpha}^{\beta} z^{\alpha} \frac{\partial}{\partial z^{\beta}}
$$

This vector field projects to a holomorphic vector field $X$ on $\mathbf{C} P^{q}$ and all holomorphic vector fields on $\mathbf{C} P^{q}$ arise this way. It is now easy to find $f_{X}$ from the formula $\bar{\partial} f_{X}=\Omega\left(X_{A},-\right)$ as follows:

$$
\Omega(X,-)=\left(a_{\alpha}^{\beta} z^{\alpha} \frac{\partial}{\partial z^{\beta}}\right) i \partial \bar{\partial} \log |z|^{2}=i \bar{\partial}\left(\frac{a_{\alpha}^{\beta} z^{\alpha} \bar{z} \beta}{|z|^{2}}\right) .
$$

So

$$
f_{X}=i \frac{a_{\alpha}^{p} z^{\alpha} \bar{z}^{\beta}}{|z|^{2}}+C_{A}
$$

where $C_{A}$ is a constant chosen so that $\int_{\mathbf{C} p^{q}}\left(-1 / f_{X}\right) \boldsymbol{\nu}=1$.

We can now investigate the holomorphic foliation associated to the section $\phi=\left(-1 / f_{X}\right) \omega \otimes X$. From the definition of $E_{\phi}$, it is easy to see 
that it is the subbundle of $T\left(S^{2 q+1}\right)^{\mathrm{C}}$ given by

$$
E_{\phi}=H^{0,1} \oplus \mathbf{C} \cdot Y_{\phi}
$$

where $H^{0,1}$ is the subspace of horizontal vectors of type $(0,1)$ and

$$
Y_{\phi}=X_{H}-f_{X} V
$$

where $X_{H}$ denotes the horizontal lift of $X$. Using the formula

$$
X_{H}=\tilde{X}_{A}-a_{\alpha}^{\beta} z^{\alpha} \bar{z}^{\beta}\left(z^{\gamma} \frac{\partial}{\partial z^{\gamma}}\right)
$$

and setting $B=\left[b_{\alpha}^{\beta}\right]=\left[a_{\alpha}^{\beta}+\left(C_{A} / q\right) \delta_{\alpha}^{\beta}\right] \in \mathrm{GL}(q+1, \mathbf{C})$ we obtain the formula

$$
Y_{\phi}=b_{\alpha}^{\beta} z^{\alpha} \frac{\partial}{\partial z^{\beta}}-\left(b_{\alpha}^{\alpha}\right)\left(\bar{z}^{\delta} \frac{\partial}{\partial \bar{z}^{\delta}}\right) .
$$

As observed in [3], the foliation $E_{\phi}$ is the pull-back to $S^{2 q+1}$ of the codimension q holomorphic foliation on $\mathbf{C}^{q+1}$ given by the (complex) integral curves of the holomorphic vector fields

$$
\tilde{X}_{B}=b_{\alpha}^{\beta} z^{\alpha} \frac{\partial}{\partial z^{\beta}} .
$$

Indeed it is easily seen from (2.13) and (2.14) that the normal bundle of $E_{\phi}$ is generated by the pull-backs to $S^{2 q+1}$ of the one forms of type $(1,0)$ annihilating $\tilde{X}_{B}$.

Denote by $\mathscr{F}_{B}$ the foliation on $S^{2 q+1}$ associated to $B$. From (2.13) we see that the complex structure on the normal bundle is in some sense kept fixed - what changes is the underlying real foliation.

2.16 Remarks. (1) Since multiplying $B$ by a scalar does not change the foliation $\mathscr{F}_{B}$, the map $B \mapsto \mathscr{F}_{B}, B \in \mathrm{SL}(q+1, \mathrm{C})$ given a parameterization of a neighborhood of the Hopf foliation by a neighborhood of the identity in $\operatorname{SL}(q+1, C)$. The above discussion shows this parameterization to be equivalent to that of $\S 1$. The complex dimension of the parameter space is $(q+1)^{2}-1$.

The above analysis can be used to give a more useful classification of holomorphic foliations near the Hopf foliation.

2.17 TheOREM. There is a neighborhood $U$ of the identity in $\mathrm{GL}(q+1, \mathrm{C})$ such that if $A, B \in U$ are two conjugate matrices, then $\mathscr{F}_{A}$ and $\mathscr{F}_{B}$ are conjugate foliations. In particular, every foliation sufficiently near the 
Hopf foliation is conjugate to one of the form $\mathscr{F}_{J}$ where

$$
J=\left|\begin{array}{llllll} 
& J_{1} & & & & 0 \\
& & J_{2} & & & \\
& & & \ddots & \\
0 & & & & J_{l}
\end{array}\right|
$$

with

$$
J_{k}=\left|\begin{array}{cccccc} 
& \lambda_{k} & & \frac{1}{4} & & 0 \\
& \cdot & & \frac{1}{4} & \\
0 & & \lambda_{k} & & & \frac{1}{4}
\end{array}\right|
$$

an $n_{k} \times n_{k}$ matrix with $\left|\lambda_{k}-1\right|<\frac{1}{4}$.

Proof. Let $B \in \mathrm{GL}(q+1, \mathrm{C})$ be so near the identity that $X_{B}\left(|z|^{2}\right)$ has positive real part for all $z \in \mathbf{C}^{q+1} \backslash\{0\}$ and suppose $B$ is conjugate to a matrix $J$ as in the statement of the theorem. Let $u=\left(u^{1}, \ldots, u^{q+1}\right)$ be coordinates relative to a basis in which $B$ assumes the form $J$ and set $f_{0}(z)=|z|^{2}$ and $f_{1}(z)=|u|^{2}$.

Construct a regular isotopy of embeddings $\phi_{t}: S^{2 q+1} \rightarrow \mathbf{C}^{q+1} \backslash\{0\}$, $0 \leq t \leq 1$ as follows. Let $f_{t}(z)$ be the quadratic form

$$
f_{t}(z)=(1-t) f_{0}(z)+t f_{1}(z)
$$

and set

$$
\phi_{t}(z)=a(t, z) \cdot z
$$

where $a(t, z)>0$ is the unique positive real number characterized by the condition $f_{t}(a(t, z) \cdot z)=1$.

Letting $\mathscr{F}_{B}$ denote the foliations on $\mathbf{C}^{q+1} \backslash\{0\}$ given by the complex integral curves of $X_{B}$, it is clear that the pull-back foliation $\phi_{0}^{*}\left(\tilde{\mathscr{F}}_{B}\right)$ is just $\mathscr{F}_{B}$. Moreover, since the image of $\phi_{1}$ is just the set $\left\{\left.z \in \mathbf{C}^{q+1}\left|f_{1}(z)=\right| u\right|^{2}\right.$ $=1\}$ the pull-back foliation $\phi_{1}^{*}\left(\tilde{\mathscr{F}}_{B}\right)$ is just $\widetilde{F}_{J}$.

We need only show that the imbeddings $\phi_{t}$ are all transversal to $\mathscr{F}_{B}$. For then $\mathscr{F}_{B}$ and $\mathscr{F}_{J}$ are isotopic to one another and therefore conjugate (H. B. Lawson, Foliations, Bull. Amer. Math. Soc. 80 (1974), 369-418). To prove that $\phi_{t}$ is transversal to $\tilde{\mathscr{F}}_{B}$ it is sufficient to show that the real part of $X_{B}\left(f_{t}\right)$ is positive at all points of $\mathbf{C}^{q+1} \backslash\{0\}$. 
But by assumption $X_{B}\left(f_{0}\right)$ has positive real part. The formula

$$
X_{B}\left(f_{1}\right)=\left(\bar{u}^{1}, \ldots, \bar{u}^{q+1}\right) \cdot J \cdot\left(\begin{array}{c}
u^{1} \\
\vdots \\
u^{q+1}
\end{array}\right),
$$

together with the inequality $\left|\lambda_{k}-1\right|<\frac{1}{4}$ for all $k$, easily yields the inequality

$$
\operatorname{Re}\left(X_{B}\left(f_{1}\right)\right)>\left(1-\frac{1}{4}\right)|u|^{2}-\frac{1}{4}|u|^{2}>0
$$

and therefore

$$
\operatorname{Re}\left(X_{B}\left(f_{t}\right)\right)=(1-t) \operatorname{Re}\left(X_{B}\left(f_{0}\right)\right)+t \operatorname{Re}\left(X_{B}\left(f_{1}\right)\right)>0 .
$$

2.18 Remarks. (1) From 2.17 and the explicit formulas for the integral curves of $X_{J}$, it follows that $\mathscr{F}_{B}$ is a Riemannian foliation if and only if $B$ is diagonalizable with eigenvalues satisfying the condition $\lambda_{k} / \lambda_{l}$ real for all $k$ and $\mathscr{F}_{B}$ is compact if in addition $\lambda_{k} / \lambda_{\text {l }}$ is rational for all $k$.

(2) Note that Theorem 2.17 gives a parameterization of conjugacy classes of holomorphic foliation near the Hopf foliations by a $(q)$-dimensional analytic space.

\section{REFERENCES}

[1] T. Duchamp and M. Kalka, Deformation theory for holomorphic foliations, J. Differential Geom., 14 (1979), 317-337.

[2] _ Stability theorems for holomorphic foliations, Trans. Amer. Math. Soc., 260 (1980), 255-266.

[3] J. Girbau, A. Haefliger and D. Sundararaman, On deformations of transversely holomorphic foliations, I.H.E.S. preprint.

[4] X. Gomez-Mont, Transversal holomorphic structures, Thesis, Princeton University (1978).

[5] K. Kodaira, L. Nirenberg and D. Spencer, On the existence of deformations of complex analytic structures, Ann. of Math., 68 (1958), 450-459.

[6] __ Differential Topology, Foliations and Gelfand-Fuks Cohomology, ed. Paul A. Schweitzer. Springer Lecture Notes 654 (1978).

Received March 18, 1981. Research of the second author was partially supported by NSF grant MCS 8001531.

THE UNIVERSITY OF WASHINGTON

SEATTLE, WA 98195

AND

TULANE UNIVERSITY

NeW ORLEANS, LA 70118 



\section{PACIFIC JOURNAL OF MATHEMATICS \\ EDITORS}

DONALD BABBITT (Managing Editor)

University of California.

Los Angeles, CA 90024

Hugo Rossi

University of Utah

Salt Lake City, UT 84112

C. C. Moore and Arthur Ogus

University of California

Berkeley, CA 94720
J. DugundjI

Department of Mathematics

University of Southern California

Los Angeles, CA 90089-1113

R. FinN and H. Samelson

Stanford University

Stanford, CA 94305

\section{ASSOCIATE EDITORS}
R. ARENS
E. F. BECKENBACH
B. H. NeumanN
F. WOLF
K. YoshidA (1906-1982)

\section{SUPPORTING INSTITUTIONS}

UNIVERSITY OF ARIZONA

UNIVERSITY OF BRITISH COLUMBIA

CALIFORNIA INSTITUTE OF TECHNOLOGY

UNIVERSITY OF CALIFORNIA

MONTANA STATE UNIVERSITY

UNIVERSITY OF NEVADA. RENO

NEW MEXICO STATE UNIVERSITY

OREGON STATE UNIVERSITY
UNIVERSITY OF OREGON

UNIVERSITY OF SOUTHERN CALIFORNIA

STANFORD UNIVERSITY

UNIVERSITY OF HAWAII

UNIVERSITY OF TOKYO

UNIVERSITY OF UTAH

WASHINGTON STATE UNIVERSITY

UNIVERSITY OF WASHINGTON 


\section{Pacific Journal of Mathematics}

Vol. 112, No. $1 \quad$ January, 1984

Richard Blaine Barrar and Henry Loeb, Characterizing the divided difference weights for extended complete Tchebycheff systems $\ldots \ldots \ldots \ldots 1$

Harold Bennett and David John Lutzer, Generalized ordered spaces with

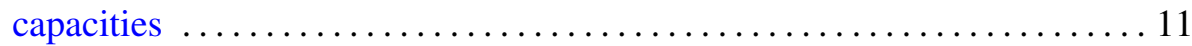

Geoffrey J. Butler and Lynn Harry Erbe, Comparison theorems for second-order operator-valued linear differential equations

Bohumil Cenkl and Richard D. Porter, de Rham theorem with cubical

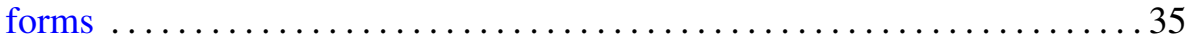

Zvonko Cerin, Characterizing global properties in inverse limits . ....... 49

Thomas Eugene Duchamp and Morris Kalka, Holomorphic foliations and deformations of the Hopf foliation .........................69 69

John Paul Hempel, Homology of coverings $\ldots \ldots \ldots \ldots \ldots \ldots \ldots \ldots \ldots$

Gerald Norman Hile and R. Z. Yeh, Inequalities for eigenvalues of the

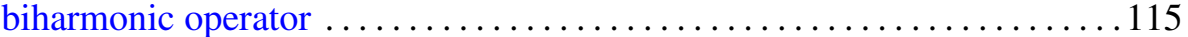

Kenneth Irwin Joy, A description of the topology on the dual space of a

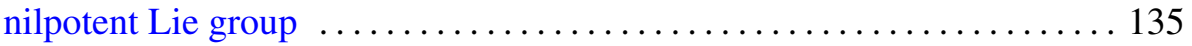

Alex Kumjian, On localizations and simple $C^{*}$-algebras $\ldots \ldots \ldots \ldots \ldots 141$

Bernardus de Pagter, The space of extended orthomorphisms in a Riesz

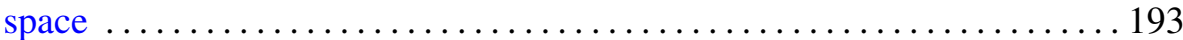

Stephen C. Persek, Iterated averaging for periodic systems with hidden

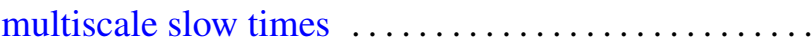

David Francis Rearick, Divisibility of arithmetic functions . . . . . . . . . 237

Masaaki Suzuki, The intrinsic metrics on the circular domains in $\mathbf{C}^{n}$ 